LAWRENCE LIVERMORE N A TIO N A L LABORATORY

\section{Laboratory Studies of the Fe K-shell Emission}

P. Beiersdorfer

December 27, 2004

X-ray Diagnostics of Astrophysical Plasmas

Cambridge, MA, United States

November 15, 2004 through November 17, 2004 
This document was prepared as an account of work sponsored by an agency of the United States Government. Neither the United States Government nor the University of California nor any of their employees, makes any warranty, express or implied, or assumes any legal liability or responsibility for the accuracy, completeness, or usefulness of any information, apparatus, product, or process disclosed, or represents that its use would not infringe privately owned rights. Reference herein to any specific commercial product, process, or service by trade name, trademark, manufacturer, or otherwise, does not necessarily constitute or imply its endorsement, recommendation, or favoring by the United States Government or the University of California. The views and opinions of authors expressed herein do not necessarily state or reflect those of the United States Government or the University of California, and shall not be used for advertising or product endorsement purposes. 


\title{
Laboratory Studies of the Fe K-shell Emission
}

\author{
P. Beiersdorfer \\ Lawrence Livermore National Laboratory, Livermore, CA 94550
}

\begin{abstract}
.
An overview is given of measurements of the Fe K-shell spectrum from low-density laboratory sources. The measurements include wavelengths, electron-impact collision cross sections, innershell ionization phenomena, dielectronic recombination resonance strengths, charge exchange recombination, electron beam polarization effects, resonance excitation, and radiative cascade contributions. $\mathrm{K}$-shell spectra have now also been obtained with microcalorimeters, including microcalorimeter arrays that are twins of the ASTRO-E and ASTRO-E2 missions, which illustrate typical resolving powers and spectral capabilities.
\end{abstract}

\section{INTRODUCTION}

The importance of the Fe K-shell emission for astrophysics has been evident for many years. Data have typically been obtained with rather low-resolution solid-state detectors such as those used on ASCA or presently on Chandra. Next year's anticipated launch of $A S T R O-E 2$ is expected to provide Fe K-shell spectra with high-resolution $(E / \Delta E \approx$ 1000). This will enable spectroscopy of the Fe $\mathrm{K}$ lines and their use as diagnostics of such source parameters as temperature, ionization equilibrium, red shift, and potentially electron density.

In order to analyze Fe K-shell data, spectral models must be both accurate and complete. Laboratory data provide tests for both. The Fe K-shell spectrum has been studied in low-density laboratory sources in much detail, i.e., on tokamaks and the Livermore electron beam ion traps. Laboratory data now include accurate line lists and identifications, electron-ion collision cross sections, innershell ionization phenomena and cross sections, dielectronic recombination resonance strengths, spectra produced by charge exchange recombination, electron beam polarization effects, resonance excitation, and radiative cascade contributions.

A review of laboratory X-ray astrophysics measurements has recently been given [1]. This review covered many measurements of the Fe K-shell emission, including measurements of non-equilibrium phenomena in ionizing plasmas, such as those found in young supernova remnants, as well as in recombining plasma, such as those found in photo-ionized sources. In the following, we will provide an overview of laboratory measurements complementary to this review. 


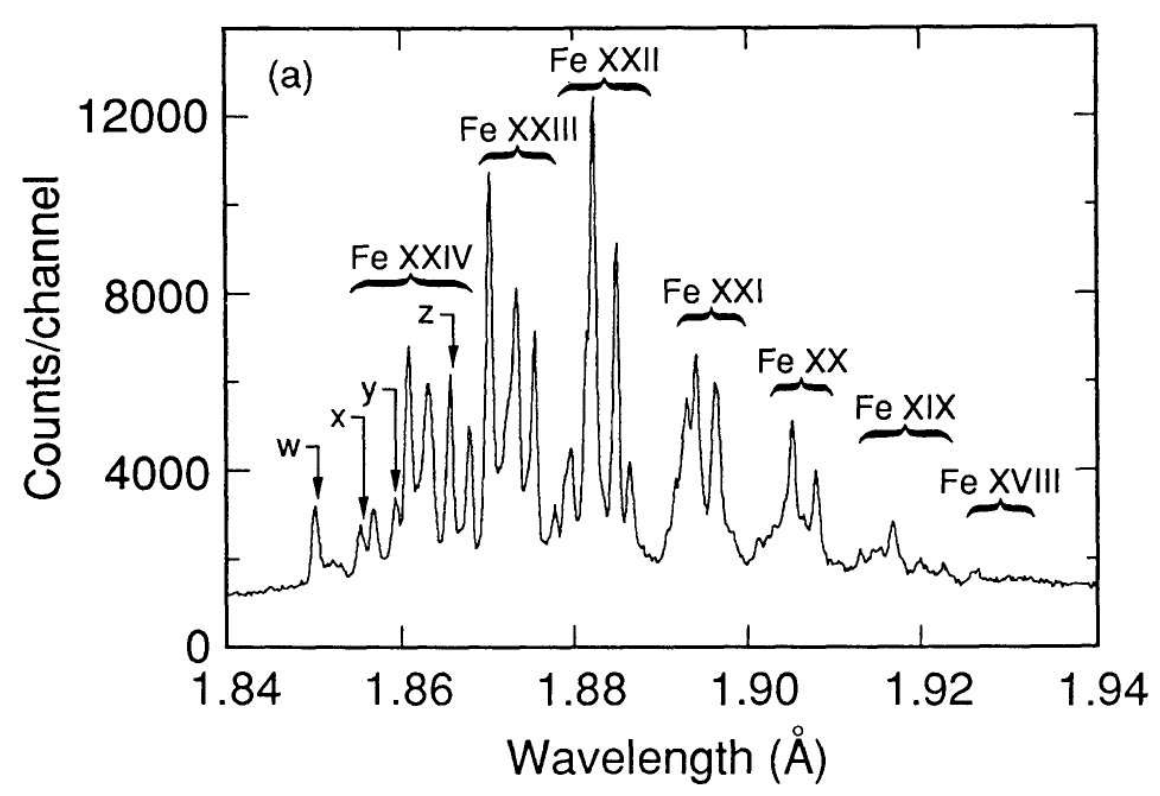

FIGURE 1. Tokamak spectrum of the Fe K-shell emission. From [3].

\section{WAVELENGTH MEASUREMENTS}

Line lists of the Fe $\mathrm{K}$ lines have been produced by detailed spectral measurements from various sources. Measurements using low-density sources include the Sun [2], the PLT tokamak [3], and the Livermore EBIT-II electron beam ion trap [4].

A spectrum of the $2 \rightarrow 1 \mathrm{Fe} \mathrm{K}$-shell emission is shown in Fig. 1. The spectrum was recorded with a crystal spectrometer on the PLT tokamak with $\leq 1.0 \mathrm{keV}$ electron temperature. At these temperatures, the spectrum is dominated by lines from Fe XXII and Fe XXIII, and lines from charge states as low as Fe XVII and as high as Fe XXV are seen.

Essentially all strong lines in the spectrum have been identified. However, there is some uncertainty concerning blends. Moreover, the emission from the intermediate charge states of iron is sensitive to the electron density for $n_{e} \geq 10^{11} \mathrm{~cm}^{-3}$, which complicates the analysis $[3,5,6]$.

Lines have also been studied for the higher members of the Fe K-shell Rydberg series. As an example we show in Fig. 2 crystal-spectrometer data of the $3 \rightarrow 1 \mathrm{Fe} \mathrm{K}$ emission recorded on the TFTR tokamak.

\section{LINE STRENGTHS}

Excitation processes of the Fe K-shell emission comprise a complex mix, as described in more detail by Jacobs et al. in these proceedings. A typical modeling spectrum of the Fe $3 \rightarrow 1$ transitions is shown in Fig. 2. The model spectrum was produced with 

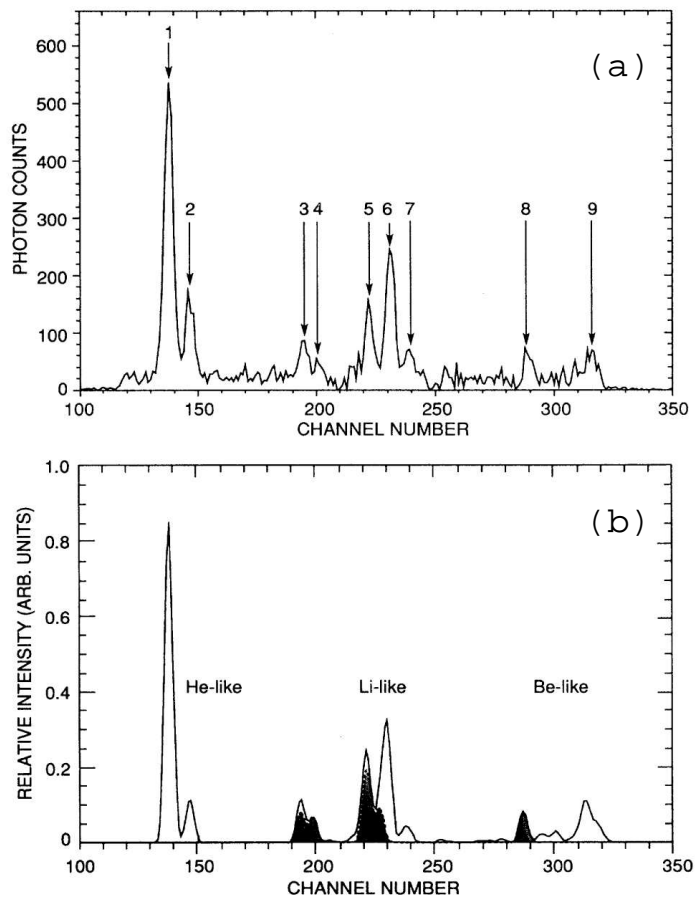

FIGURE 2. Spectrum of the Fe $3 \rightarrow 1 \mathrm{~K}$-shell emission: (a) Tokamak data; (b) collisional radiative modelling calculations. From [7].

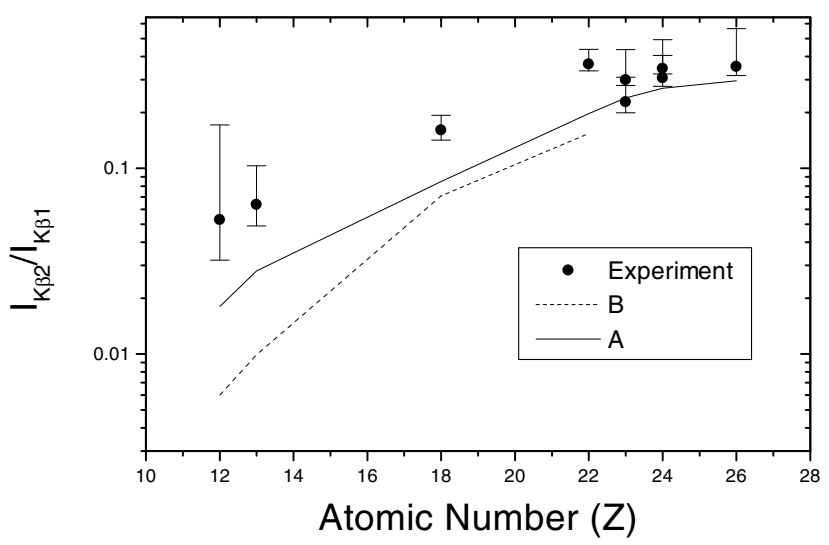

FIGURE 3. Comparison of the ratio of the intensities of the $3 \rightarrow 1$ intercombination and resonance lines in various heliumlike ions with two predictions. From [7].

the HULLAC package of atomic codes [8] and generally reproduces well the observed emission.

A noteable difference between the tokamak spectrum and the HULLAC model can be seen in the size of the $1 s 3 p^{3} P_{1} \rightarrow 1 s^{2}{ }^{1} S_{0}$ intercombination line in Fe XXV (labeled 


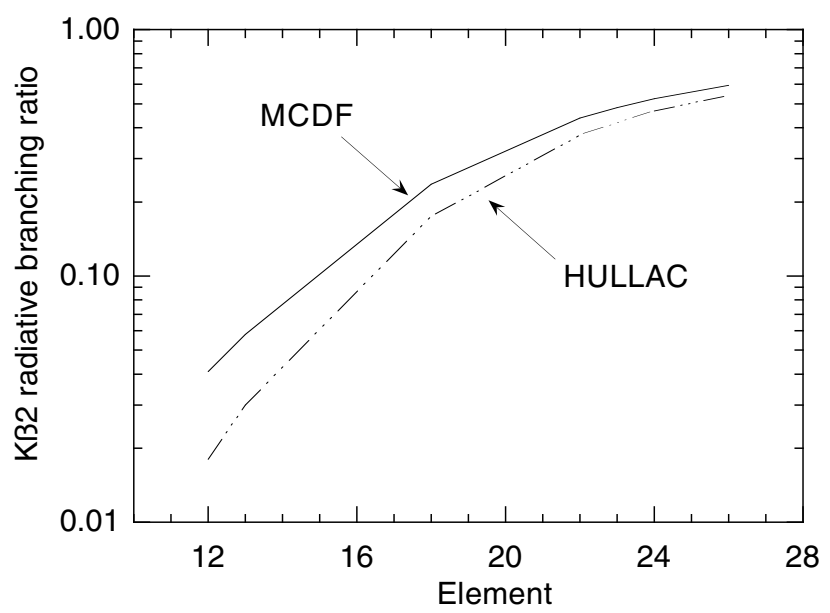

FIGURE 4. Comparison of the radiative branching ratio affecting the $3 \rightarrow 1$ intercombination line emanating from the $1 s 3 p^{3} P_{1}$ upper level in various heliumlike ions. From [10].

1 in Fig. 2) relative to the $1 s 3 p{ }^{1} P_{1} \rightarrow 1 s^{2}{ }^{1} S_{0}$ resonance line (labeled 2 in Fig. 2). The HULLAC model predicts a ratio that is about a factor of two less than observed [7, 9].

This ratio was investigated further using the ion traps at Livermore [10]. The measurements found a systematic underestimation of this ratio by modeling calculations, as illustrated in Fig. 3. In part this discrepancy was attributed to inaccuracies in the calculation of the radiative branching ratio that affects the strength of the intercombination line. The radiative branching ratio calculated by HULLAC is systematically smaller than those obtained with a multi-configuration Dirac-Fock (MCDF) calculation [10], as shown in Fig. 4. However, even correcting for the branching ratio, calculations do not yet match the laboratory ratios, as shown by the solid line in Fig. 3.

\section{DIELECTRONIC RECOMBINATION}

The K-shell Fe spectrum contains many lines produced exclusively by dielectronic recombination [11]. While many lines are well resolved, others blend with some of the strongest spectral lines. For example, dielectronic satellite transitions overlap with the Fe XXV $1 s 2 p^{1} P_{1} \rightarrow 1 s^{2}{ }^{1} S_{0}$ resonance line $w$ and can significantly change its apparent intensity, especially at low temperature [12].

Using the EBIT-II electron beam ion trap at Livermore the emission from dielectronic satellite lines $1 s 2 p n \ell \rightarrow 1 s^{2} n \ell$ with $n \geq 3$ in the vicinity of line $w$ were measured using a high-resolution crystal spectrometer [13], as shown in Fig. 5. The measured resonance strengths agree with calculations at the 10-20\% level [1]. 


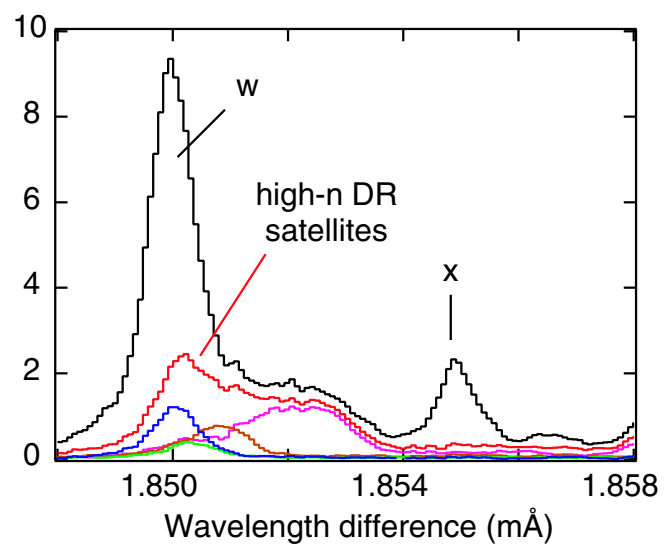

FIGURE 5. Contributions from high-n satellites $(n \geq 3)$ to the Fe XXV resonance line $w$. From [13].

\section{CHARGE EXCHANGE}

Charge exchange between neutral gases and highly charged ions is a very effective process. Charge exchange cross sections are on the order of $10^{-15} \mathrm{~cm}^{2}$, which is a value many orders of magnitude larger than those for electron-impact excitation - Fe K-shell excitation cross sections are on the order of $10^{-22} \mathrm{~cm}^{2}$ [14]. As a result, charge exchange may dominate the line formation processes in those cases where neutral species are present, even at a very low fraction.

$\mathrm{K}$-shell X-ray production via charge exchange has been studied using the Livermore electron beam ion traps [16, 17]. A recent spectrum [15] of the K-shell emission from Fe XXV produced in the reaction

$$
\mathrm{Fe}^{25+}+A \rightarrow \mathrm{Fe}^{24+*}+A^{+}
$$

is shown in Fig. 6. Here $A$ represents a neutral species, $N_{2}$ in the present case. The emission is compared with the emission by electron-impact excitation. The two spectra, taken with a low-resolution solid-state detector, look very similar. However, the centroid of the $2 \rightarrow 1$ emission produced by charge exchange is shifted by more than $20 \mathrm{eV}$ from that produced by electron impact excitation, indicating that the spectral details have changed markedly [15].

In order to investigate the spectral details of charge exchange produced line emission, high-resolution crystal spectrometer measurements were made of the K-shell spectrum of Ar XVII on the NSTX tokamak [18]. As intimated by the Fe XXV measurements, the relative intensity of the resonance line $w$, intercombination lines $x$ and $y$, and forbidden line $z$ in the Ar XVII is very different than expected from spectra recorded from collisional plasmas. In particular, the resonance line $w$ is weaker than the other three lines. This is reminiscent of the $\mathrm{K}$-shell Fe XXV excited by radiative recombination, i.e., by the capture of a free electron by an $\mathrm{Fe}^{25+}$ ion [1]. 

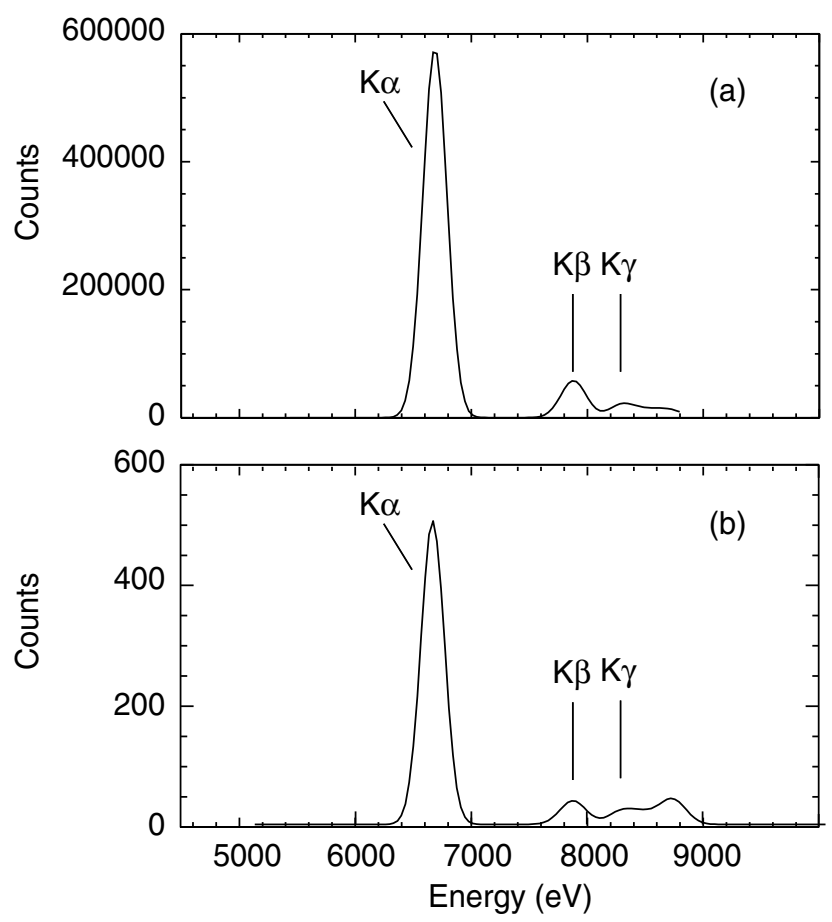

FIGURE 6. Fe XXV K-shell emission: (a) formed by electron-impact collisions; (b) formed by charge exchange of neutral gases with $\mathrm{Fe}^{25+}$ ions. From [15].

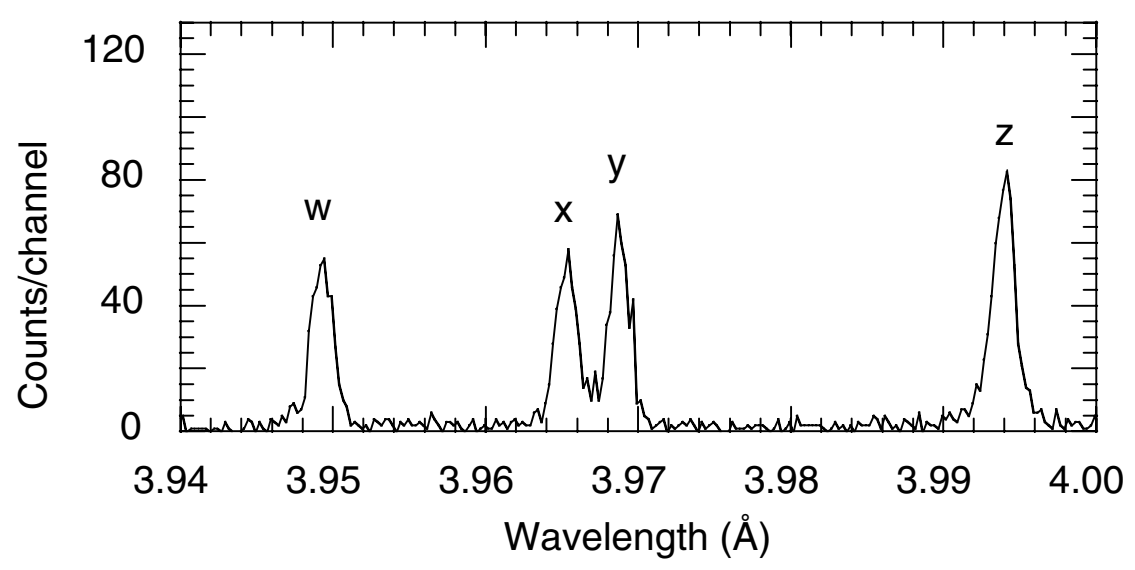

FIGURE 7. Spectrum of the Ar XVII K-shell emission produced by charge exchange in the reaction $A r^{17+}+H \rightarrow A r^{16+*}+H^{+}$. From [18].

\section{MICROCALORIMETER MEASUREMENTS}

The use of microcalorimeters for X-ray spectroscopy opens a new era of astrophysics. The first microcalorimeter measurement of the Fe K emission was carried out on the Livermore EBIT-II electron beam ion trap using a Livermore microcalorimeter [19]. That measurement achieved a resolution of about $20-25 \mathrm{eV}$. The best spectrum of the measurement series, selected for the highest apparent resolution, is shown in Fig. 8(a). 

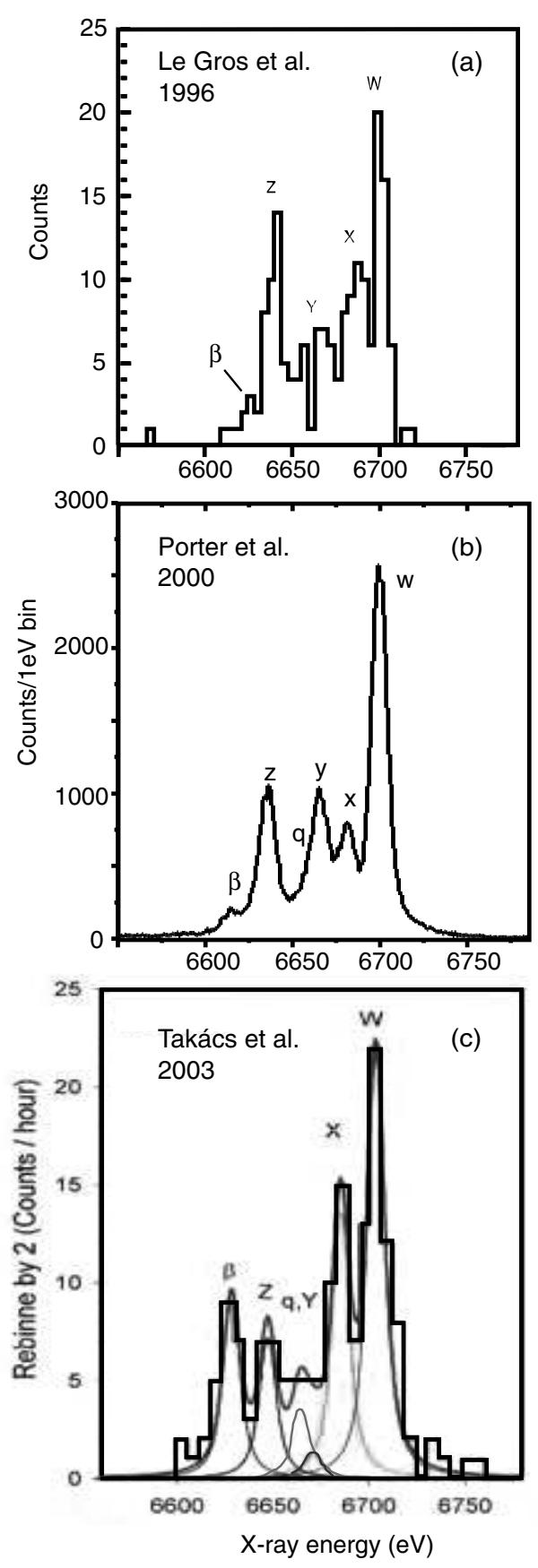

FIGURE 8. Spectrum of the Fe K-shell emission recorded with a microcalorimeter: (a) single-picel Livermore calorimeter (from [19]); (b) 32-pixel GSFC calorimeter (from [20]); (c) single-pixel SAO calorimeter (from [21]).

Upon relocation to the Smithsonian Astrophysical Observatory and further development, the instrument was used to record an Fe XXV spectrum on the electron beam ion trap at NIST [22, 21] (see Fig. 8(c)). A resolution of $6 \mathrm{eV}$ was now attributed to the instrument [22]. Precision microcalorimeter instruments have been developed at the Goddard Space 


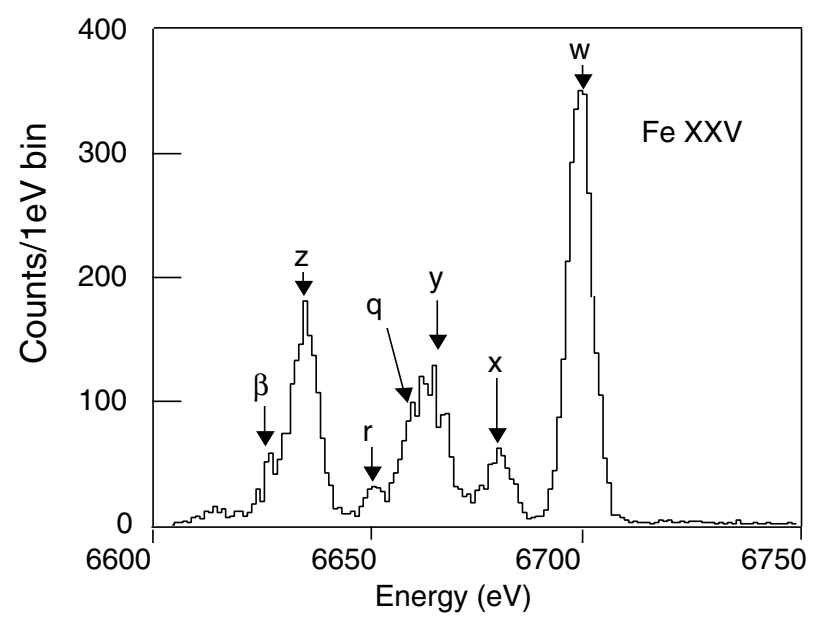

FIGURE 9. Microcalorimeter spectrum of the Fe K-shell emission from EBIT-I using the twin XRS from ASTRO-E2. From [25].

Flight Center as part of the ASTRO-E and ASTRO-E2 programs. A measurement of the $\mathrm{Fe} X X V \mathrm{~K}$ lines performed with a twin detector of the ASTRO-E mission is shown in Fig. 8(b). This measurement was carried out on the Livermore EBIT-I electron beam ion trap [20]. A resolution of $11 \mathrm{eV}$ was attributed to this spectrum. A unique combination of long-term gain stability and high effective area enabled the high statistics measurement shown.

Microcalorimeters represent new X-ray instrumentation, and the data they produce have to measure up to decades of data produced by crystal spectrometers (which generally still have higher resolving powers than calorimeters). In this context it is interesting to note that the intercombination line $x$ is larger than $y$ in (a) and (c). The effect is grossly exaggerated in (c), where $x$ is the second strongest line in the spectrum. Such a large ratio of $x$ to $w$ cannot be consistently explained by atomic physics, especially when considering that line $y$ is nearly absent in this spectrum. By contrast, the intensity ratio of $x$ to $w$ and $x$ to $y$ in spectrum (b) is as expected from crystal spectrometer measurements, e.g. $[14,23]$.

It is also interesting to note that the resonance line $\beta$ in Fe XXIII, situated on the low-energy side of the Fe XXV forbidden line $z$, is large in the measurement carried out on the NIST electron beam ion trap (cf. Fig. 8(c)). In fact, it is the third largest line in the spectrum. From the spectrum and the fits provided by Takács et al. [21] we derive an abundance ratio of $0.40: 0.20: 1.00$ for Fe XXIII : Fe XXIV : Fe XXV. In other words, there is more Fe XXIII and Fe XXV than Fe XXIV. Such a hollow abundance ratio is atypical for an electron beam ion trap. By contrast, $\beta$ is small in the measurements carried on the Livermore electron beam ion traps (cf. Fig. 8(a) and (b)). This is consistent with a charge balance that peaks at Fe XXV. Indeed, from the spectrum in (b) we derive an abundance ratio of $0.07: 0.22: 1.00$ for Fe XXIII : Fe XXIV : Fe XXV. Such a charge balance is sensible for ion trap plasmas and in accordance with abundance measurements carried out with crystal spectrometers [24].

As illustrated by our discussion, the ASTRO-E microcalorimeter generally matches 


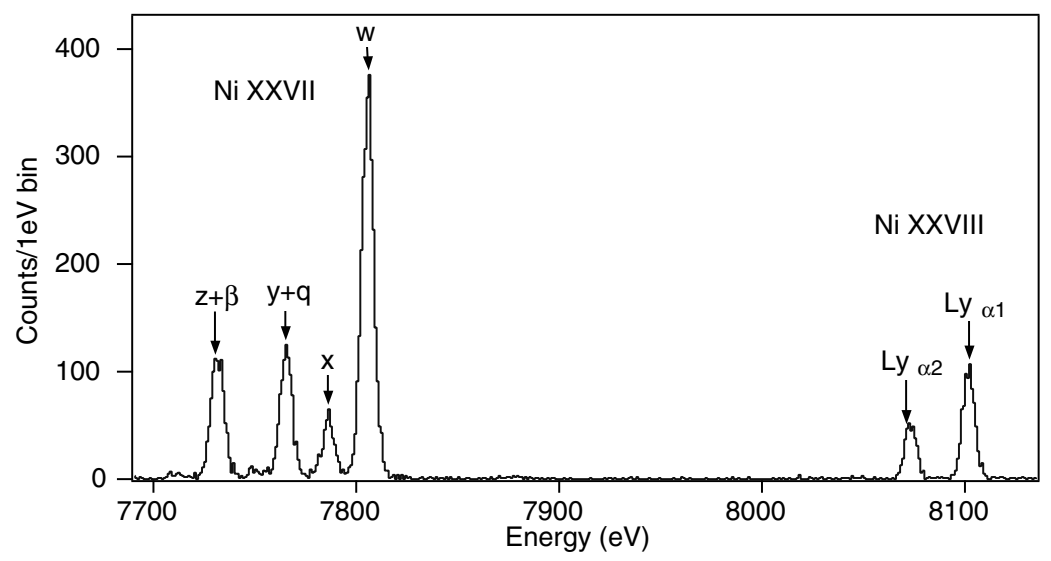

FIGURE 10. Microcalorimeter spectrum of the Ni K-shell emission from EBIT-I using the twin XRS from ASTRO-E2. From [26].

the expectations set by the multitude of crystal spectrometer measurements and has been found to have high spectral fidelity.

The ASTRO-E calorimeter has now been superceded by the ASTRO-E2 calorimeter. Its twin X-ray Spectrometer (XRS) was installed at the Livermore EBIT-I electron beam ion trap in October 2003. A spectrum of the Fe $\mathrm{K}$ emission is shown in Fig. 9. The ASTROE2 microcalorimeter has an improved resolution compared to the ASTRO-E calorimeter. This is evident when comparing the spectrum in Fig. 9 with that in Fig. 8(b). The instrument has a resolution of $6 \mathrm{eV}$ [25]. Although $6 \mathrm{eV}$ is the same resolution assigned to the spectrum in Fig. 8(c) by Silver et al., a comparison shows that the ASTRO-E2 microcalorimeter clearly resolves lines in a superior fashion. In Fig. 10 we show the K-shell spectrum of Ni recorded with the ASTRO-E2 XRS on EBIT-I [26].

Like its predecessor, the ASTRO-E2 microcalorimeter has high spectral fidelity and matches laboratory measurements carried out with proven x-ray instrumentation. In particular, the ratios of $x$ and $y, x$ and $w$, etc. agree well with those recorded with crystal spectrometers, as does the inferred abundance ratio of Fe XXIII, Fe XXIV and Fe XXV. This bodes very well for the capabilities of the ASTRO-E2 mission.

\section{CONCLUSIONS}

The Fe $\mathrm{K}$ spectrum has been studied in great detail, and much laboratory data are available for anchoring spectral modeling codes. Nevertheless, it still offers surprises, and new results are expected to come forth in the near future, especially pertaining to line formation by charge exchange and microcalorimeter measurements.

\section{ACKNOWLEDGMENTS}

The author wishes to thank F. Scott Porter and Bradford Wargelin for making results available before publication. This work was performed under the auspices of the U.S. 
DOE by the University of California Lawrence Livermore National Laboratory under contract W-7405-Eng-48 and support by NASA's Astronomy and Physics Research and Analysis Program under contract S-06553-G.

\section{REFERENCES}

1. Beiersdorfer, P., Ann. Rev. Astron. Astrophys., 412, 343 (2003).

2. Seely, J. F., Feldman, U., and Safronova, U. I., Astrophys. J., 304, 838 (1986).

3. Beiersdorfer, P., Phillips, T., Jacobs, V. L., Hill, K. W., Bitter, M., von Goeler, S., and Kahn, S. M., Astrophys. J., 409, 846 (1993).

4. Decaux, V., Jacobs, V. L., Beiersdorfer, P., Liedahl, D. A., and Kahn, S. M., Phys. Rev. A, 68, 012509 (2003).

5. J. R. Lemen, K. J. H. Phillips, R. D. Cowan, and I. P. Grant, Astron. Astrophys., 135, 313 (1984).

6. Jacobs, V. L., Doschek, G. A., Seely, J. F., and Cowan, R. D., Phys. Rev. A, 39, 2411 (1989).

7. Smith, A. J., Bitter, M., Hsuan, H., Hill, K. W., von Goeler, S., Timberlake, J., Beiersdorfer, P., and Osterheld, A., Phys. Rev. A, 47, 3073 (1993).

8. Bar-Shalom, A., Klapisch, M., and Oreg, J., J. Quant. Spectrosc. Rad. Transfer, 71, 179 (2001).

9. Beiersdorfer, P., Osterheld, A. L., Phillips, T. W., Bitter, M., Hill, K. W., and von Goeler, S., Phys. Rev. E, 52, 1980 (1995).

10. A.J. Smith, P. Beiersdorfer, K.J. Reed, A.L. Osterheld, V. Decaux, K. Widmann, and M.H. Chen, Phys. Rev. A, 62, 012704 (2000).

11. Gabriel, A. H., Mon. Not. R. Astron. Soc., 160, 99 (1972).

12. Bitter, M., von Goeler, S., Hill, K. W., Horton, R., Johnson, D., Roney, W., Sauthoff, N., Silver, E., and Stodiek, W., Phys. Rev. Lett., 47, 921 (1981).

13. Beiersdorfer, P., Schneider, M. B., Bitter, M., and von Goeler, S., Rev. Sci. Instrum., 63, 5029 (1992).

14. Wong, K. L., Beiersdorfer, P., Reed, K. J., and Vogel, D. A., Phys. Rev. A, 51, 1214 (1995).

15. B. J. Wargelin et al., in preparation.

16. Beiersdorfer, P., Olson, R. E., Brown, G. V., Chen, H., Harris, C. L., Neill, P. A., Schweikhard, L., Utter, S. B., and Widmann, K., Phys. Rev. Lett., 85, 5090 (2000).

17. Beiersdorfer, P., Lisse, C. M., Olson, R. E., Brown, G. V., and Chen, H., Astrophys. J. (Lett.), 549, L147 (2001).

18. Beiersdorfer, P., Bitter, M., Marion, M., and Olson, R. E., in preparation.

19. Le Gros, M., Silver, E., Beiersdorfer, P., López-Urrutia, J. C., Widmann, K., and Kahn, S. M., in Electron Beam Ion Trap Annual Report 1995, University of California Lawrence Livermore National Laboratory Report UCRL-ID-124429 (Livermore, CA), p.22 (1996).

20. Porter, F. S., Audley, M. D., Beiersdorfer, P., Boyce, K. R., Brekosky, R. P., Brown, G. V., Gendreau, K. C., Gygax, J., Kahn, S., Kelley, R. L., Stahle, C. K., and Szymkowiak, A. E., Proc. SPIE, 4140, 407 (2000).

21. Takács, E., Silver, E., Laming, J. M., Gillaspy, J. D., Schnopper, H., Brickhouse, N., Barbera, M., Mantraga, M., Ratliff, L. P., Tawara, H., Makónyi, K., Madden, N., Landis, D., Beeman, J., and Jaller, E. E., Nucl. Instrum. Methods Phys. Res. B, 205, 144 (2003).

22. Silver, E., Schnopper, H., Bandler, S., Brickhouse, N., Murray, S., Barbera, M., Takács, E., Gillaspy, J. D., Porto, J. V., Kink, I., Ratliff, P. R., Hudson, L., Laming, J. M., Madden, N., Landis, D., Beeman, J., Haller, E. E., and Schuch, R., in Photonic, Electronic \& Atomic Collisions, ed. by J. Burgdörfer and J. S Cohen and S. Datz and C. R. Vane (Rinton Press, Princeton), p. 312 (2003).

23. Bitter, M., von Goeler, S., Horton, R., Goldman, M., Hill, K., Sauthoff, N. R., and Stodiek, W., Phys. Rev. Lett., 42, 304 (1979).

24. Wong, K., Beiersdorfer, P., Marrs, R. E., Penetrante, B. M., Reed, K. J., Scofi eld, J. H., Vogel, D. A., and Zasadzinski, R., Nucl. Instrum. Methods, B72, 234 (1992).

25. F. S. Porter et al., in preparation.

26. Porter, F. S., Brown, G. V., Boyce, K. R., Kelley, R. L., Kilbourne, C. A., Beiersdorfer, P., Chen, H., Terracol, S., Kahn, S. M., and Szymkowiak, A. E., Rev. Sci. Instrum., 75, 3772 (2004). 